\section{Perfil e processo da assistência prestada ao recém-nascido de risco no Sul do Brasil}

\section{Profile and process of the care provided to high-risk newborns in southern Brazil}

Willian Augusto de Melo'

Taqueco Teruya Uchimura"

'Departamento de Enfermagem do Centro Universitário de Maringá (CESUMAR), Maringá-PR.

"Programa de Pós-Graduação Mestrado em Enfermagem da Universidade Estadual de Maringá, Maringá, PR.

\section{Resumo}

Objetivo: Caracterizar os recém-nascidos (RN) de risco e verificar o processo de assistência dispensado pelo Programa de Vigilância ao Recém-nascido de Risco do município de Maringá, PR. Metodologia: Os dados foram coletados dos prontuários e das Fichas de Acompanhamento de uma amostragem estratificada composta por 505 $\mathrm{RN}$ de risco nascidos em 2007. As variáveis maternas, neonatais e assistenciais foram analisadas descritivamente utilizando o software Statistica 7.1. Resultados: O Programa considerou somente os riscos biológicos como critério de inclusão, podendo os mesmos estar isolados $(63,2 \%)$ ou associados entre si (36,8\%). Sobre as mães, $71,5 \%$ eram adultas, $78,2 \%$ com escolaridade $\geq 8$ anos de estudo, $57,2 \%$ sem companheiro, $55,3 \%$ com ocupação não remunerada, $69,5 \%$ com $\geq 6$ consultas pré-natal, $87,3 \%$ de gravidez única e $65,4 \%$ realizaram parto cesáreo. Sobre os RN de risco, 51\% eram masculinos, $50,3 \%$ com baixo peso ao nascer, $51,5 \%$ a termo, 95,8\% sem anomalias congênitas e 90,3\% com Apgar $\geq 7$ no $5^{\circ}$ minuto. Sobre a assistência prestada às crianças de risco 69,5\% foram acompanhadas pelo Programa, $71 \%$ dos prontuários foram localizados, $82,6 \%$ com nenhuma visita domiciliar, $8,9 \%$ receberam $\geq 12$ consultas médicas, $33,1 \%$ não receberam nenhuma orientação, 5,8\% foram hospitalizados, $18,7 \%$ apresentaram $\geq$ 12 pesagens, e $19,8 \%$ apresentaram registro de imunização completa. Conclusão: Fazse necessário reorganizar a atenção básica que assegure a integralidade da assistência e à continuidade do acompanhamento do desenvolvimento e crescimento biopsicossocial da criança de risco.

Palavras-chave: Recém-nascido. Grupos de risco. Assistência integral à saúde da criança. Assistência de enfermagem. Assistência médica. Serviços de Vigilância Epidemiológica. 


\section{Abstract}

Objective: To describe newborns at risk and check the process of care provided by the High Risk Newborn Surveillance Program in Maringá-PR. Methods: Data were collected from medical records and monitoring sheets of a stratified sample consisting of 505 newborns at risk, born in 2007. Maternal and neonatal care were analyzed descriptively using Statistica 7.1 software. Results: The program only considered biological risks as inclusion criteria, whether they appeared alone $(63.2 \%)$ or associated (36.8\%). Regarding mothers, $71.5 \%$ were adults, $78.2 \%$ had $\geq 8$ years of schooling, $57.2 \%$ were single parents, $55.3 \%$ had an unpaid occupation, $69.5 \%$ had $\geq 6$ prenatal visits, $87.3 \%$ had a single baby, and $65.4 \%$ had a C-section. Regarding newborns at risk, $51 \%$ were male, $50.3 \%$ had low birth weight, $51.5 \%$ were full term, $95.8 \%$ without congenital anomalies, and $90.3 \%$ with $5^{\text {th }}$ minute scores $\geq 7$. Regarding care provided to children at risk, $69.5 \%$ were accompanied by the Program, $71 \%$ of the medical records were located, for $82.6 \%$ there were no home visits, $8.9 \%$ received $\geq$ 12 medical consultations, $33.1 \%$ had not received any guidance, $5.8 \%$ were hospitalized, $18.7 \%$ were weighed $\geq 12$ times, and $19.8 \%$ presented full immunization records. Conclusion: It is necessary to reorganize primary care to ensure integral care and for ongoing monitoring of the bio-psychosocial development and growth of children at risk.

Keywords: Infant, Newborn. Risk Groups. Comprehensive Health Care. Nursing care. Medical Care. Epidemiologic Surveillance Services.

\section{Introdução}

Apesar de o avanço tecnológico possibilitar melhora na sobrevida das crianças menores de um ano de idade, os índices de morbidade e mortalidade infantil ainda são estatisticamente significativos e preocupantes $^{1}$. Neste sentido, o modelo de vigilância à saúde, proposto para crianças que apresentam um maior risco de morbidade e mortalidade, tem sido classificado por diversos estudos como risco biológico e risco social ${ }^{2-3}$.

Dentre os riscos biológicos, estão aqueles relacionados com as condições da gravidez da mãe e do nascimento do $\mathrm{RN}$, tais como baixo peso ao nascer, prematuridade, malformação congênita, índice de Apgar no quinto minuto $<7$, mãe com sorologia positiva para o Vírus da Imunodeficiência Humana (HIV) ou outras doenças transmissíveis e mãe portadora de doença mental ${ }^{4,5}$.

Para os riscos sociais, destacam-se a mãe menor de 18 anos, mãe analfabeta, parto extra-hospitalar, número de irmãos vivos maior ou igual a três, número de irmãos mortos maior ou igual a um, chefe da família desempregado, mãe sem companheiro, residente em área de risco (bairros periféricos listados na ficha de notificação e que têm em comum a urbanização precária pela falta de pavimentação, o esgoto a céu aberto e as dificuldades de acesso aos serviços de saúde pela distância e pela precariedade dos meios de transporte coletivo), mãe sem seguimento pré-natal e dependência química do pai ou mãe ao álcool, ao fumo e às drogas lícitas e ilícitas ${ }^{2-3}$.

Assim, com o intuito de reduzir a mortalidade infantil, o Ministério da Saúde (MS) recomenda garantir o acompanhamento do RN de risco após a alta do hospital, por meio da implementação do Programa de Vigilância ao RN de Risco (PVRNR) ${ }^{6}$.

O PVRNR propõe um acompanhamento ambulatorial para que se assegure a continuidade da assistência ao bebê e sua família; a avaliação do equilíbrio psicoafetivo entre o bebê e sua família; detecção e intervenção nas situações de risco como 
ganho de peso inadequado, sinais de refluxo gastroesofágico, infecções, apnéias, entre outros $^{6}$, assegurando que o bebê receba toda a assistência necessária, respeitando em todos os momentos os vínculos entre a mãe e o bebê ${ }^{6}$.

O MS afirma que a responsabilidade das Equipes de Saúde da Família (ESF) se mantém sobre a população de crianças de risco, prevendo acompanhamento mensal, controle de peso, altura, avaliação clínica, participação em grupos educativos e orientação nutricional ${ }^{4}$.

As crianças de risco devem ser priorizadas para o desenvolvimento das ações de vigilância à saúde, na captação precoce $\mathrm{e}$ busca ativa para a manutenção do calendário de atenção à saúde da criança e no cumprimento da proposta de acompanhamento do seu crescimento e desenvolvimento ${ }^{2,6}$.

Assim sendo, este estudo teve como objetivo traçar o perfil sociodemográfico, materno e neonatal, além de verificar o processo de assistência dispensado ao RN de risco pelo PVRNR do município de Maringá, PR.

\section{Metodologia}

Trata-se de um estudo transversal descritivo-exploratório com abordagem quantitativa. Faz parte do projeto aprovado pelo Conselho Nacional de Desenvolvimento Científico e Tecnológico (CNPq) no edital $n^{\circ} 15 / 2007$, coordenado pelo Departamento de Enfermagem da Universidade Estadual de Maringá (UEM).

O PVRNR foi criado pela Secretaria Municipal de Saúde do município de Maringá, PR, no ano de 2000, com objetivo de acompanhar todas as crianças nascidas visando à identificação e à intervenção nos fatores de risco que influenciam na morbimortalidade infantil ${ }^{7}$. Os critérios de inclusão que definiram o ingresso dos $\mathrm{RN}$ de risco no PVRNR no município de Maringá foram: idade materna menor ou igual a 17 anos, peso ao nascer menor que $2.500 \mathrm{~g}$, idade gestacional menor de 37 semanas, valor do Apgar no $5^{\circ}$ minuto menor ou igual a 7 , pre- sença de anomalia congênita, mãe HIV(+) e outros critérios considerados relevantes ou graves ${ }^{7}$. A Secretaria Municipal de Saúde de Maringá preconiza que seria responsabilidade de cada UBS o acompanhamento da criança até completar um ano de vida, garantindo-lhe uma consulta ao mês e VD quando houvesse necessidade de busca ativa aos faltosos? ${ }^{7}$.

Para seleção das crianças optou-se pela amostragem aleatória com erro padrão amostral de $1 \%$, com objetivo de compensar as eventuais perdas. Do total de 4.175 nascimentos, 710 foram inseridos no PVRNR. Foram selecionados 505 RN para composição da amostra que foi estratificada proporcionalmente conforme o número de crianças cadastradas em cada uma das 25 Unidades Básicas de Saúde (UBS). Ressaltase que fizeram parte da amostra as crianças nascidas no período de 2007, pois o PVRNR preconiza acompanhar da criança de risco por 12 meses após o seu nascimento ${ }^{7}$.

Com relação às fontes de dados, utilizaram-se o SINASC para verificação, comparação e posterior linkage com outros bancos de dados construídos posteriormente, o prontuário da criança na UBS que continha dados referentes ao processo da assistência à criança do nascimento até o primeiro ano de vida, as Fichas de Acompanhamento e de Avaliação da Alta que continham informações sobre os dados da mãe, das condições de nascimento, critérios de inclusão, informações sobre o acompanhamento mensal da criança provenientes das consultas médicas e de enfermagem e os dados sobre o tipo de alta que a criança recebe do Programa ao completar 12 meses de idade, podendo ser classificada como saudável, mudança de endereço, inclusão em programas, óbito, não avaliado ou outros. Os dados foram coletados no período de março a julho de 2009.

As variáveis estudadas foram categorizadas considerando-se o ponto de corte preconizado pelo Ministério da Saúde para risco ou não risco ${ }^{4,5}$.

Para as variáveis maternas considerouse idade ( $<18$ e $\geq 18$ anos), escolaridade $(<$ $8 \mathrm{e} \geq 8$ anos de estudo), estado civil (sem $\mathrm{e}$ 
com companheiro), ocupação (remunerada e não), número de consultas pré-natal $(\leq 6$ e $>6$ ), tipo de gravidez (múltipla e única) e tipo de parto (cesário e vaginal).

As variáveis relacionadas ao $\mathrm{RN}$ de risco foram: sexo (masculino e feminino), peso ao nascer ( $<2500$ e $\geq 2500 \mathrm{~g}$ ), estatura ao nascer $(<47 \mathrm{e} \geq 47 \mathrm{~cm})$, idade gestacional $(<37$ e $\geq 37$ semanas), Apgar no $1^{\circ}$ e $5^{\circ}$ minuto ( $\leq 7$ e $>7$ ), raça/cor (não branca e branca), anomalia congênita (sim e não) e critério de risco (associado e isolado).

As variáveis relacionadas à assistência prestada à criança de risco foram: número de visita domiciliar, encaminhamentos, hospitalizações, orientações ofertadas, legibilidade, assinatura dos registros. Para as variáveis atendimentos médicos e de enfermagem, número de pesagens e medições de comprimento, seguiu-se as preconizações adotadas pelo PVRNR, adotando-se 12 atendimentos como ou número considerado adequado (ponto de corte). Ressalta-se que para a variável imunização foram considerados sete registros de comparecimentos à UBS como ponto de corte, por ser o número ideal para que a criança complete seu esquema vacinal durante o primeiro ano de vida conforme preconização do Ministério da Saúde ${ }^{8}$. Salienta-se que o ponto de corte utilizado pelo PVRNR para Apgar $(\leq 7)$ diferia da preconização do MS $(<7)$.

Foi realizada a análise descritiva das tabelas de frequências, com distribuições percentuais para as variáveis qualitativas e com o cálculo de medidas de tendência central como médias, medianas e desvios padrão para as variáveis quantitativas. Os dados coletados foram arquivados no Programa Excel e, para a análise univariada das variáveis, foi utilizado o software Statística 7.1.

O estudo obedeceu aos princípios éticos, sendo aprovado mediante o Parecer 069/2009 do Comitê Permanente de Ética em Pesquisa Envolvendo Seres Humanos da Universidade Estadual de Maringá, e foi dispensado do Termo de Consentimento Livre e Esclarecido por se tratar de dados originários de fontes secundárias.

\section{Resultados}

Quanto aos critérios de inclusão, o PVRNR considerou os riscos biológicos relacionados à mãe e ao $\mathrm{RN}$, podendo os mesmos estar isolados ou associados entre si (Tabela 1).

A idade materna foi o critério isolado mais prevalente $(36,0 \%)$, seguido de baixo peso ao nascer e prematuridade (Tabela 1). Dos RN que apresentaram dois critérios de inclusão associados surgiram 11 subgrupos com seus pares de critérios sendo predominante o subgrupo do baixo peso ao nascer e a prematuridade $(79,2 \%)$. A presença de três critérios de inclusão ou mais ocorreu em menor proporção com sete subgrupos, sendo que o subgrupo prevalente foi o do baixo peso ao nascer, prematuridade e Apgar $\leq 7$ no quinto minuto (Tabela 1 ).

Com relação à idade materna dos $\mathrm{RN}$ de risco, a média encontrada foi de $24,2 \pm 7,3$ anos, a mediana 23 e a moda 17 anos, sendo a mínima encontrada de 13 e a máxima de 47 anos (Tabela 2).

Observou-se prevalência das mães que possuíam oito anos ou mais de estudo enquanto que, no estado marital, predominaram as mães sem companheiro, considerando-se como parte deste grupo as solteiras, as viúvas e as separadas judicialmente (Tabela 2 ).

Com relação às atividades ocupacionais, houve prevalência para aquelas que apresentavam atividade não remunerada, pertencentes às categorias "dona de casa" e "estudantes" (Tabela 2).

Com relação às variáveis neonatais, encontrou-se número ligeiramente maior de $\mathrm{RN}$ de risco do sexo feminino (Tabela 3).

Em relação ao peso ao nascer, evidenciou-se que 254 (50,3\%) dos $\mathrm{RN}$ de risco apresentaram baixo ao peso $(<2.500 \mathrm{~g})$ (Tabela 3). A prematuridade foi verificada em 250 (49,5\%) dos RN estudados e para a variável raça/cor houve forte predominância da raça branca comparada com a não branca (Tabela 3).

Dos tipos de anomalias congênitas encontradas, a fenda labial e palatina e as 
Tabela 1 - Distribuição do número e percentagem de recém-nascidos de risco segundo os critérios de inclusão do PVRNR. Maringá, PR, 2007.

Table 1 - Distribution of the number and percentage of newborns at risk according to the inclusion criteria of PVRNR. Maringá, PR, 2007.

\begin{tabular}{|c|c|c|}
\hline Critérios de Inclusão & $\mathrm{N}$ & $\%$ \\
\hline \multicolumn{3}{|l|}{ Critérios de inclusão isolados } \\
\hline Idade materna $<18$ anos & 115 & 36,0 \\
\hline Peso $<2500 \mathrm{~g}$ & 92 & 28,8 \\
\hline Idade gestacional $<37$ semanas & 72 & 22,6 \\
\hline Apgar $5^{\circ} \min \leq 7$ & 19 & 6,0 \\
\hline Presença de anomalia & 14 & 4,4 \\
\hline Outros (Mãe HIV+, síflis e toxoplasmose) & 7 & 2,2 \\
\hline Total & 319 & 63,2 \\
\hline \multicolumn{3}{|l|}{ Dois critérios de inclusão associados } \\
\hline Idade materna/Peso & 6 & 3,8 \\
\hline Idade materna/Idade gestacional & 10 & 6,3 \\
\hline Idade materna/Apgar & 2 & 1,3 \\
\hline Idade materna/Anomalia & 1 & 0,6 \\
\hline Idade materna/Outros & 1 & 0,6 \\
\hline Peso/ldade gestacional & 126 & 79,2 \\
\hline Peso/Apgar & 5 & 3,1 \\
\hline Idade gestacional/Apgar & 5 & 3,1 \\
\hline Idade gestacional/Outros & 1 & 0,6 \\
\hline Apgar/Anomalia & 1 & 0,6 \\
\hline Presença de anomalia/Outros & 1 & 0,6 \\
\hline Total & 159 & 31,4 \\
\hline \multicolumn{3}{|l|}{ Três critérios de inclusão associados ou mais } \\
\hline Idade materna/Peso/ldade gestacional & 6 & 22,2 \\
\hline Idade materna/Idade gestacional/Apgar & 1 & 3,7 \\
\hline Peso/Idade gestacional/Apgar & 16 & 59,3 \\
\hline Peso/Idade gestacional/Anomalia & 1 & 3,7 \\
\hline Peso/Idade gestacional/Outros & 1 & 3,7 \\
\hline Idade materna/Peso/ldade gestacional/Apgar & 1 & 3,7 \\
\hline Anomalia/Peso/ldade gestacional/Apgar & 1 & 3,7 \\
\hline Total & 27 & 5,4 \\
\hline
\end{tabular}

malformações do sistema nervoso apresentaram seis casos para cada tipo $(28,6 \%)$, seguidas das deformações congênitas do aparelho osteomuscular com quatro (19\%), anomalia cromossômica com dois (9,5\%), e onfacelite e as malformações do aparelho circulatório todas com um caso para cada tipo (4,8\%).

A Tabela 4 demonstra a assistência dispensada ao $\mathrm{RN}$ de risco pela equipe multiprofissional, salientando que 359 (71\%) das crianças tiveram seus prontuários localizados nas UBS.

Constatou-se que, ao longo do primeiro ano de vida, a maioria das crianças não recebeu visita domiciliar (VD), ter recebido apenas uma VD constituiu-se a moda (10,5\%) e o número máximo de VD recebido foi oito (Tabela 4).

Em relação aos atendimentos médicos e de enfermagem dispensados aos $\mathrm{RN}$ de risco notou-se uma divergência inversamente proporcional entre seus dois extremos no número destas ocorrências. Dos RN que não receberam qualquer atendimento, a maior proporção $(22,8 \%)$ foi por falta de atendimento médico, enquanto a menor $(6,7 \%)$ foi por falta de atendimento da 
Tabela 2 - Distribuição do número e percentagem dos RN de risco segundo as variáveis maternas, Maringá, PR, 2007.

Table 2 - Distribution of the number and percentage of newborns at risk according to the maternal variables. Maringá, PR, 2007.

\begin{tabular}{|c|c|c|}
\hline Variáveis Sociodemográficas Maternas & $\mathrm{N}$ & $\%$ \\
\hline \multicolumn{3}{|l|}{ Idade Materna } \\
\hline$<18$ & 144 & 28,5 \\
\hline$\geq 18$ a 34 & 308 & 61,0 \\
\hline$\geq 35$ & 53 & 10,5 \\
\hline \multicolumn{3}{|l|}{ Escolaridade (anos de estudo) } \\
\hline Analfabeta & 1 & 0,2 \\
\hline 1 a 3 & 5 & 1,0 \\
\hline 4 a 7 & 104 & 20,6 \\
\hline 8 a 11 & 316 & 62,5 \\
\hline 12 e mais & 79 & 15,7 \\
\hline \multicolumn{3}{|l|}{ Estado Civil } \\
\hline Solteira & 286 & 56,6 \\
\hline Casada/União consensual & 216 & 42,8 \\
\hline Viúva & 1 & 0,2 \\
\hline Separada & 2 & 0,4 \\
\hline \multicolumn{3}{|l|}{ Ocupação } \\
\hline Dirigentes de organizações & 13 & 2,6 \\
\hline Profissionais das ciências e artes & 39 & 7,7 \\
\hline Serviços administrativos & 32 & 6,3 \\
\hline Técnicos de nível médio & 9 & 1,8 \\
\hline Vendedores do comércio em lojas e mercados & 92 & 18,2 \\
\hline Produção de bens e serviços industriais & 39 & 7,7 \\
\hline Manutenção e reparação & 2 & 0,4 \\
\hline Estudante* & 63 & 12,5 \\
\hline Dona de casa* & 216 & 42,8 \\
\hline \multicolumn{3}{|l|}{ Pré-Natal (No de Consultas) } \\
\hline Nenhuma & 9 & 1,8 \\
\hline 1 a 3 & 26 & 5,1 \\
\hline 4 a 6 & 119 & 23,6 \\
\hline 7 e mais & 351 & 69,5 \\
\hline \multicolumn{3}{|l|}{ Tipo de Gravidez } \\
\hline Única & 441 & 87,3 \\
\hline Múltipla & 64 & 12,7 \\
\hline \multicolumn{3}{|l|}{ Tipo de Parto } \\
\hline Vaginal & 175 & 34,6 \\
\hline Cesária & 330 & 65,4 \\
\hline Total & 505 & 100 \\
\hline
\end{tabular}

* Ocupação não remunerada. Occupation unpaid.

enfermagem, e dos RN que receberam 12 ou mais atendimentos, a menor proporção $(8,9 \%)$ foi por atendimento médico enquanto a maior $(30,1 \%)$ foi por atendimento da enfermagem (Tabela 4). Observou-se que a proporção de crianças não atendidas por profissional médico foi três vezes maior que a proporção não atendida pela equipe de enfermagem.

Sobre a quantidade de orientações recebidas prevaleceram as orientações relacionadas ao desenvolvimento psicomotor seguidas daquelas crianças que não receberam qualquer orientação (Tabela 4). 
Tabela 3 - Distribuição do número e percentagem dos RN de risco segundo as variáveis neonatais. Maringá, PR, 2007.

Table 3 - Distribution of the number and percentage of newborns at risk according to neonatal variables. Maringá, PR, 2007.

\begin{tabular}{|c|c|c|}
\hline Variáveis Neonatais & $\mathrm{N}$ & $\%$ \\
\hline \multicolumn{3}{|l|}{ Sexo } \\
\hline Masculino & 248 & 49,1 \\
\hline Feminino & 257 & 50,9 \\
\hline \multicolumn{3}{|l|}{ Peso ao nascer } \\
\hline$<1500 \mathrm{~g}$ & 33 & 6,5 \\
\hline 1500 a $2499 g$ & 221 & 43,8 \\
\hline 2500 a $3999 g$ & 243 & 48,1 \\
\hline$\geq 4000 \mathrm{~g}$ & 8 & 1,6 \\
\hline \multicolumn{3}{|l|}{ Estatura (cm) } \\
\hline$<47$ & 84 & 16,7 \\
\hline$\geq 47$ & 72 & 14,2 \\
\hline Sem informação & 349 & 69,1 \\
\hline \multicolumn{3}{|c|}{ Idade Gestacional (Semanas) } \\
\hline 22 a 27 & 6 & 1,2 \\
\hline 28 a 31 & 33 & 6,5 \\
\hline 32 a 36 & 211 & 41,8 \\
\hline 37 a 41 & 250 & 49,5 \\
\hline$\geq 42$ & 5 & 1,0 \\
\hline \multicolumn{3}{|l|}{ Apgar $1^{\circ} \mathrm{min}$} \\
\hline$\leq 7$ & 143 & 28,3 \\
\hline$>7$ & 362 & 71,7 \\
\hline \multicolumn{3}{|l|}{ Apgar $5^{\circ} \mathrm{min}$} \\
\hline$\leq 7$ & 49 & 9,7 \\
\hline$>7$ & 456 & 90,3 \\
\hline \multicolumn{3}{|l|}{ Raça/Cor } \\
\hline Branco & 467 & 92,5 \\
\hline Preto & 5 & 1,0 \\
\hline Amarelo & 2 & 0,4 \\
\hline Pardo & 31 & 6,1 \\
\hline \multicolumn{3}{|l|}{ Anomalia congênita } \\
\hline Sim & 21 & 4,2 \\
\hline Não & 484 & 95,8 \\
\hline Total & 505 & 100 \\
\hline
\end{tabular}

Verificou-se a realização de 2.512 registros de pesagens e 2.363 registros de medições de comprimento durante o período, sendo que entre as crianças que apresentaram frequência maior que 12 destas práticas os registros foram semelhantes tanto para pesagem quanto para medição de comprimento (Tabela 5).

Percebeu-se que a frequência dos procedimentos antropométricos foi insuficiente, não cumprindo a meta do Programa, pois o ideal (12 pesagens e 12 medições do comprimento durante o primeiro ano de vida) para as 359 crianças seria de 4.308 para cada medida, porém o encontrado correspondeu a $2.512(58,3 \%)$ pesagens e $2.363(54,8 \%)$ medições de comprimentos (Tabela 5). A média de realização destas medidas por criança variou de $7 \pm 5,3$ para a pesagem e 6,5 \pm 5,3 para medição de comprimento. O valor mínimo e a moda foi zero e 27 o valor máximo encontrado de pesagem e de 
Tabela 4 - Distribuição do número e percentagem dos procedimentos assistenciais ao RN de risco. Maringá, PR, 2007.

Table 4 - Distribution of the number and percentage of care procedures to the newborns at risk. Maringá, $P R, 2007$.

\begin{tabular}{|c|c|c|}
\hline Variáveis assistenciais & $\mathrm{N}$ & $\%$ \\
\hline \multicolumn{3}{|l|}{ Visita domiciliar } \\
\hline Nenhuma & 417 & 82,6 \\
\hline 1 a 3 & 80 & 15,8 \\
\hline 4 a 7 & 5 & 1,0 \\
\hline $8 \mathrm{e}+$ & 3 & 0,6 \\
\hline Total* & 505 & 100 \\
\hline \multicolumn{3}{|l|}{ Atendimentos médicos } \\
\hline Nenhum & 82 & 22,8 \\
\hline 1 a 3 & 94 & 26,2 \\
\hline 4 a 7 & 95 & 26,5 \\
\hline 8 a 11 & 56 & 15,6 \\
\hline 12 ou + & 32 & 8,9 \\
\hline Total** & 359 & 100 \\
\hline \multicolumn{3}{|l|}{ Atendimentos enfermagem } \\
\hline Nenhum & 24 & 6,7 \\
\hline 1 a 3 & 44 & 12,3 \\
\hline 4 a 7 & 95 & 26,5 \\
\hline 8 a 11 & 88 & 24,5 \\
\hline $12 \mathrm{ou}+$ & 108 & 30,1 \\
\hline Total** & 359 & 100 \\
\hline \multicolumn{3}{|l|}{ Orientações recebidas } \\
\hline Nenhuma & 119 & 23,5 \\
\hline Higienização & 23 & 4,5 \\
\hline Imunização & 25 & 4,9 \\
\hline Aleitamento materno & 26 & 5,1 \\
\hline Alimentação & 29 & 5,7 \\
\hline Desenvolvimento psicomotor & 195 & 38,5 \\
\hline Outras & 16 & 3,2 \\
\hline Sem definição por extenso & 74 & 14,6 \\
\hline Total ${ }^{* * *}$ & 507 & 100 \\
\hline \multicolumn{3}{|l|}{ Destino dos encaminhamentos } \\
\hline Hospital Municipal & 74 & 20,3 \\
\hline Hospital Universitário & 6 & 1,6 \\
\hline Saúde bucal & 12 & 3,3 \\
\hline Outras UBS & 20 & 5,5 \\
\hline Não encaminhado & 252 & 69,2 \\
\hline Total*** & 364 & 100 \\
\hline \multicolumn{3}{|l|}{ Hospitalização } \\
\hline Sim & 21 & 5,8 \\
\hline Não & 338 & 94,2 \\
\hline Total** & 359 & 100 \\
\hline
\end{tabular}

* Dados da Ficha de Acompanhamento do PVRNR ( $=505)$. Data Sheet Monitoring of the PVRNR $(N=505)$.

** Prontuários localizados $(\mathrm{N}=359)$. Records located $(\mathrm{N}=359$.)

*** Algumas crianças receberam mais que uma orientação/encaminhamento $(\mathrm{N}=359)$. Some children received more than one orientation/referral. 
Tabela 5 - Distribuição do número e percentagem dos procedimentos assistenciais ao RN de risco relacionados à antropometria, imunização e qualidade dos registros. Maringá, PR, 2007.

Tables 5 - Distribution of the number and percentage of care procedures to newborns at risk related to anthropometry, immunization and quality of records. Maringá, PR, 2007.

\begin{tabular}{|c|c|c|}
\hline Assistência na antropometria e imunização & $\mathrm{N}$ & $\%$ \\
\hline \multicolumn{3}{|l|}{ Número de pesagens } \\
\hline Nenhum & 36 & 10,0 \\
\hline 1 a 4 & 100 & 27,9 \\
\hline 5 a 8 & 91 & 25,3 \\
\hline 9 a 11 & 65 & 18,1 \\
\hline$\geq 12$ & 67 & 18,7 \\
\hline \multicolumn{3}{|l|}{ Legibilidade registros de pesagem } \\
\hline Todos legíveis & 311 & 86,6 \\
\hline$\geq 1$ llegível & 6 & 1,7 \\
\hline Não registrado & 36 & 10 \\
\hline Ignorado & 6 & 1,7 \\
\hline \multicolumn{3}{|l|}{ Número de medições do comprimento } \\
\hline Nenhum & 49 & 13,6 \\
\hline 1 a 4 & 98 & 27,3 \\
\hline 5 a 8 & 94 & 26,2 \\
\hline 9 a 11 & 54 & 15,0 \\
\hline$\geq 12$ & 64 & 17,8 \\
\hline \multicolumn{3}{|l|}{ Legibilidade dos registros de comprimento } \\
\hline Todos legíveis & 299 & 83,3 \\
\hline$\geq 1$ llegível & 6 & 1,7 \\
\hline Não registrado & 49 & 13,6 \\
\hline Ignorado & 5 & 1,4 \\
\hline \multicolumn{3}{|l|}{ Assinatura dos registros antropométricos } \\
\hline Todos assinados & 217 & 60,4 \\
\hline$\geq 1$ não assinado & 90 & 25,1 \\
\hline Não Registrados & 39 & 10,9 \\
\hline Não Assinados & 13 & 3,6 \\
\hline \multicolumn{3}{|l|}{ № comparecimentos para imunização } \\
\hline Nenhum & 22 & 6,1 \\
\hline 1 a 3 & 67 & 18,6 \\
\hline 4 a 6 & 199 & 55,4 \\
\hline 7 ou + & 71 & 19,8 \\
\hline \multicolumn{3}{|l|}{ Legibilidade registros de imunização } \\
\hline Todos legíveis & 327 & 91,1 \\
\hline$\geq 1$ llegível & 10 & 2,8 \\
\hline Não registrado & 22 & 6,1 \\
\hline \multicolumn{3}{|l|}{ Assinatura registros imunização } \\
\hline Todos assinados & 305 & 85 \\
\hline$\geq 1$ não assinado & 23 & 6,4 \\
\hline Não Registrados & 22 & 6,1 \\
\hline Não Assinados & 9 & 2,5 \\
\hline Total & 359 & 100 \\
\hline
\end{tabular}


medições de comprimento realizadas em uma criança.

Constatou-se que os procedimentos antropométricos foram realizados e registrados por profissionais médicos e pela equipe de enfermagem com frequência equiparada para ambas as categorias e ao se verificar a qualidade destes registros esta apontou média de $85 \%$ de legibilidade (Tabela 5).Constatou-se também que 28,7\% dos prontuários que continham as medidas antropométricas registradas estavam sem as respectivas assinaturas (Tabela 5).

Enquanto a maioria das crianças, 266 (74\%), apresentou registros de comparecimento inadequados ou ausentes, observaram-se melhores resultados da qualidade destes registros quanto à legibilidade e a presença de assinatura dos profissionais (Tabela 5).

\section{Discussão}

O presente estudo encontrou elevados índices de não conformidade no processo assistencial para o RN inserido no PVRNR, principalmente nos procedimentos programados mensalmente, como os atendimentos médicos e de enfermagem, as visitas domiciliares e as orientações sobre promoção e prevenção à saúde da criança de risco.

Com relação aos critérios de inclusão no PVRNR, o percentual de mães adolescentes menores de 18 anos neste grupo (28,5\%) indica índice elevado de inclusão de RN de risco quando comparado com os demais critérios de inclusão. Estudo realizado nos Estados Unidos para determinar a faixa etária para gravidez precoce de adolescentes verificou que no grupo materno menor de 16 anos houve maior frequência de baixo peso ao nascer, prematuridade e mortalidade infantil quando comparado com as adolescentes $\geq 16$ anos, sugerindo ser esta idade o ponto de corte adequado para que a adolescente fosse considerada de risco ${ }^{9}$.

Com relação à escolaridade materna, $57,2 \%$ apresentaram $\geq 8$ anos de estudo (Tabela 2). Outros estudos que avaliaram a assistência prestada ao RN de risco encontraram proporções menores. No município de Porto Alegre, RS, em 2003, verificou-se $31,2 \%{ }^{10}$ de mães que apresentaram $\geq 8$ anos de estudo; no mesmo Estado, no município de Rio Grande, em 2005, encontrou-se $47,5 \%^{11}$. Já em Guarapuava, PR, no ano de 2005 encontrou-se índice de $51,1 \%^{3}$, e em 200,8 no município de Botucatu, SP, 36\% ${ }^{3}$.

Com relação à escolaridade, a taxa de alfabetização de adultos no município de Maringá, PR, foi de 94,6\%, e a taxa bruta de frequência escolar de $92,2 \%{ }^{12}$. Vale ressaltar ainda que, segundo o Programa das Nações Unidas para o Desenvolvimento (PNUD), o Índice de Desenvolvimento Humano (IDH) para educação no ano 2000 alcançou valor de 0,840 , considerando-se que quanto mais próximo de 1 melhor é a qualidade ${ }^{13}$.

Com relação ao estado marital, a proporção de mães sem companheiros apresentou-se elevado ao comparar com estudos semelhantes realizado no município de Rio Grande, RS, que encontrou $16,4 \%$ de mães sem companheiros ${ }^{11} \mathrm{e}$ em Santos, SP, $37,6 \%^{14}$. Sugere-se que as mães sem companheiros podem encontrar dificuldades no processo de assistência à saúde da criança, pois os estudos salientam que a presença do companheiro designa fator de proteção ${ }^{11}$.

Observando dados oficiais do Brasil, também nota-se diferença dos resultados obtidos quando comparados com o presente estudo. As informações do Relatório Final do PNDS $^{15}$ apontam que $64 \%$ das mulheres se encontraram em união estável $(36,7 \%$ formal e 27,3\% informal), sendo apenas $25,8 \%$ solteiras. Em relação às mulheres unidas formalmente, nota-se um crescimento gradativo à medida que a idade aumenta. Verificou-se também o aumento do número de viúvas, separadas, divorciadas e desquitadas ${ }^{15}$.

Estudos têm revelado a influência da ocupação materna na saúde infantil, bem como a precariedade do trabalho de algumas categorias profissionais, que nem sempre obedecem à legalidade trabalhista ${ }^{16}$. Neste estudo observou-se que a maioria das mães, 279 (55,3\%), não possuía emprego 
remunerado, sugerindo um maior tempo disponível para o filho, descartando o risco de presença da associação entre situação de risco na saúde da criança com a qualidade dos cuidados maternos oferecidos, dados confirmados em estudo realizado em Salvador, que também revelou associação com a ocupação materna, fazendo supor que a pouca disponibilidade de tempo em casa também influencia na qualidade dos cuidados maternos e, consequentemente, na assistência à saúde para essas crianças ${ }^{17}$.

O número de consultas de pré-natal ( $\geq 6$ ) demonstrou-se satisfatório quando comparado com estudo realizados em São Luis do Maranhão, que verificou a inadequação do pré-natal atingindo $49,2 \%$ e que mulheres atendidas em serviços públicos de saúde, de baixa escolaridade e baixa renda familiar, sem companheiro ou com doença durante a gravidez, tiveram maiores percentuais de inadequação ${ }^{18}$. Estudo realizado em Maringá, PR, avaliou a atenção pré-natal e demonstrou resultado superior aos parâmetros nacionais recomendados, pois verificou que as consultas de pré-natal foram garantidas, com número médio de 9,8 consultas por gestante ${ }^{19}$.

O percentual encontrado de $12,7 \%$ de gravidezes múltiplas entre os $\mathrm{RN}$ de risco é considerado alto se comparado com o total de nascimentos gemelares do município de Maringá que, em 2007, foi de $2,6 \%{ }^{20} \mathrm{im}$ plicando necessidade de maior qualidade à saúde preventiva para esta população específica. Estudo semelhante realizado no sul do país, além de encontrar índice elevado de $8,5 \%$ aponta que a gemeralidade está diretamente relacionada ao baixo peso ao nascer ${ }^{10}$. Estudo recente no município de Londrina apontou forte significância estatística $(p<0,000)$ entre nascimentos de gestações múltiplas e prematuridade ${ }^{21}$. Estudo realizado em Maringá, PR, no ano de 2008, revelou que os neonatos provenientes de gestações múltiplas apresentaram um risco 6,86 vezes maior de morrer do que os neonatos de gestações únicas, confirmando a associação $(p<0,001)$ entre o tipo de gravidez e óbitos neonatais ${ }^{1}$.
Destacou-se no presente estudo o elevado número de cesarianas $(65,4 \%)$ (Tabela 4) quando comparado a dois estudos realizados em Botucatu-SP nos anos de 2006 e 2008, relacionados ao Programa de Vigilância ao RN de Risco daquele município, encontrando uma proporção de cesarianas de $33,7 \%$ e $37,3 \%$ respectivamente $^{3,22}$. No município de Santos, SP, em estudo que também analisou o Programa de $\mathrm{RN}$ de Risco, houve $39 \%$ de cesarianas ${ }^{14}$. Estudo realizado no município de Maringá no ano de $1995^{23}$ apresentou proporções de $66,5 \%$ de cesariana em relação à duração da gestação, em neonatos pré-termo e 78,7\% em neonatos a termo, confirmando um histórico de índices elevados de cesarianas no município. Para a OMS, a taxa de cesárea deve estar entre 10 a $15 \%$ do total de partos ${ }^{24}$; porém, de acordo com as estatísticas mais recentes sobre a proporção de cesarianas, $\mathrm{o}$ Brasil apresentou no ano de 2005 um índice de $44,2 \%$, o Estado do Paraná $50,45 \%$ e a região metropolitana de Maringá $70,79 \%^{20}$.

Estudos asseguram que a cesariana exerce efeito protetor sobre a mortalidade neonatal, sobretudo nas gestações de risco, o que influencia indiretamente na assistência ao RN de risco ${ }^{25}$. Outra explicação sugerida seria que, quando detectadas precocemente, as gestações classificadas como de risco e as complicações surgidas no momento do nascimento são eventos que poderão levar o profissional obstetra a optar pela cesariana ao invés do parto normal como medida profilática para assegurar a integridade do binômio mãe e filho. Acredita-se que esta medida faz aumentar o número de cesarianas, principalmente para o grupo de RN de risco procedente ou não de uma gravidez de risco.

Embora houvesse maior proporção de RN de risco do sexo feminino, estudos semelhantes verificaram prevalência para o sexo masculino $0^{3,10,21,22}$.

A prevalência de baixo peso ao nascer pode ser considerada elevada quando comparada a outros estudos similares. No município de Porto Alegre, RS, a prevalência foi de $34,3 \%^{10}$, e no interior do Estado de 
São Paulo, $31,6 \%^{3}$. No ano de $2007,8,8 \%$ dos nascimentos ocorridos em Maringá, $\mathrm{PR}$, apresentaram baixo peso ${ }^{20}$. $\mathrm{O}$ baixo peso ao nascer está fortemente associado com a prematuridade e com o baixo número de consultas pré-natal, conforme demonstram diversos estudos sobre fatores de risco relacionados ao baixo peso ao nascer ${ }^{1,11}$. No município de Rio Grande, RS, estudo encontrou $60,7 \%$ de baixo peso ao nascer entre as crianças internadas em unidade de terapia intensiva neonatal, e 78,6\% dos óbitos neonatais ocorreram neste grupo ${ }^{11}$.

Quanto à estatura ressalta-se que foi a variável que apresentou maior índice de sub-registros (69,1\%), embora a evolução do comprimento na infância não seja preditor da estatura na idade adulta e não se associa a atrasos no desenvolvimento; por isso, a análise da estatura é dispensável para esta finalidade ${ }^{26}$.

Com relação à idade gestacional, estudos semelhantes relacionados ao $\mathrm{RN}$ de risco encontraram proporções semelhantes de prematuros com a do presente estudo (49,5\%), como no município de Rio Grande, RS, com $49,2 \%{ }^{11}$ e Porto Alegre-RS com $47,3 \%$ dos casos de prematuros dentro dos seus respectivos Programas destinados à vigilância do $\mathrm{RN}$ de risco ${ }^{10}$. Outro estudo revelou associação estatisticamente significativa entre prematuridade com alterações de desenvolvimento em crianças de alto risco e a mortalidade neonatal ${ }^{1}$.

O índice de Apgar $\leq 7$ observado em $28,3 \%$ dos RN no primeiro minuto e $9,7 \%$ no quinto (Tabela 5), foram semelhantes aos encontrados em estudo de Botucatu, SP, sendo $21,4 \%$ no primeiro minuto e $7,8 \%$ no quinto minuto ${ }^{3}$. Estudo realizado no Rio de Janeiro, RJ, revelou associação do índice de Apgar baixo com os seguintes fatores de risco: antecedente de natimorto ameaça de parto prematuro, caracterizada pela existência de contrações uterinas não típicas de trabalho de parto, baixo peso do RN inferior a $2.500 \mathrm{~g}$, e antecedente de cesariana ${ }^{27}$.

Considera-se a proporção de anomalia congênita elevada $(4,2 \%)$ (Tabela 3 ) quando comparado com o estudo realizado em
Botucatu, SP, que constatou $2 \%^{3}$. Estudo que investigou os fatores de risco para a mortalidade neonatal no município de Maringá, PR, detectou 16,1\% de anomalias congênitas ocupando estas o segundo lugar entre as causas básicas do óbito mais frequente, precedida apenas pelas afecções originadas no período perinatal $(82,1 \%)$, e observou que há um risco 23,06 vezes maior de óbito nos neonatos com malformações congênitas após se verificar a associação entre a presença de malformação congênita e a mortalidade neonatal ${ }^{1}$.

Estudos sobre a atenção dispensada ao $\mathrm{RN}$ de risco afirmam que algumas variáveis, principalmente as variáveis neonatais $\mathrm{e}$ assistenciais, podem exercer consequências clínicas negativas para o RN durante o seu primeiro ano de vida, requerendo, assim, uma atenção especializada e qualificada durante o seu processo assistencial em todos os níveis de atenção à saúde $11,14,17,19,21,25$.

Considerando que as crianças faltosas às consultas médicas devem receberVD para a busca ativa ${ }^{4}$, verificou-se que essa assistência não foi concretizada conforme preconização do Ministério da Saúde. Destaca-se que, na Estratégia de Saúde da Família (ESF), o profissional médico também executa a atividade de VD e, de acordo com o PVRNR, o número ideal de atendimentos médicos é 12 e somente $8,9 \%$ das crianças obtiveram 12 ou mais atendimentos, demonstrando que 91,1\% necessitariam receber VD para suprir estas faltas (Tabela 3).

As crianças de risco devem ser priorizadas para o desenvolvimento das ações de vigilância à saúde, na captação precoce e busca ativa para a manutenção do calendário de atenção à saúde da criança e no cumprimento da proposta de acompanhamento do seu crescimento e desenvolvimento ${ }^{4}$.

Com relação aos atendimentos médicos, melhores resultados foram verificados em estudo realizado na região metropolitana de São Paulo, onde cerca de $80 \%$ das crianças menores de um ano eram acompanhadas em algum serviço de saúde, e aproximadamente $50 \%$ com atendimento eventual, isto é, consulta não marcada ${ }^{28}$. 
A coordenação do PVRNR preconiza que a UBS é responsável pelo acompanhamento da criança durante o primeiro ano de vida, garantindo uma consulta mensal e visitas domiciliares quando se julgar necessário, além da busca ativa para os faltosos ${ }^{7}$.

Considerando que as orientações são ações fundamentais realizadas pelos profissionais de saúde para a prevenção de agravos e promoção das ações essenciais para a manutenção da saúde principalmente àqueles grupos considerados mais vulneráveis ou pertencentes a uma situação de risco $^{4}$, verificou-se pouca satisfatoriedade ao observar proporção reduzida de orientações efetuadas ao comparar com o número de crianças e atendimentos médicos e de enfermagem prestados (Tabela 4).

Com relação aos encaminhamentos e hospitalizações levou-se em consideração o sistema de referência e contra-referência, pois se acredita que este esteja intimamente ligado às questões de acessibilidade, universalidade e integralidade da assistência, que é um dos pontos importantes para viabilizar a implantação do SUS, uma vez que é a partir da sua estruturação que o encaminhamento de pacientes aos diversos níveis de atenção se torna possível. A opção pelo serviço privado para hospitalização sugere que as famílias considerem maior resolutividade para os casos julgados mais graves de alteração da saúde da criança. Nesta mesma percepção, um estudo realizado na região metropolitana de São Paulo ${ }^{31}$ verificou que as UBS foram mais utilizadas para vacinação $(97,4 \%)$ e acompanhamento de saúde (79,0\%), e os outros serviços de saúde para consultas não marcadas, com maior procura por serviços privados/convênios.

Os resultados relacionados à antropometria foram inadequados, apresentando semelhanças quando comparados ao estudo realizado no Estado de Pernambuco no ano de 2008 que, ao analisar as ações de acompanhamento do crescimento de crianças menores de um ano, revelou que aproximadamente a metade das crianças estudadas foram pesadas e apenas $21 \%$ tiveram seu comprimento medido ${ }^{29}$. A antro- pometria é apontada como sendo o melhor parâmetro para avaliar o estado nutricional com o acompanhamento sistemático do crescimento e do desenvolvimento infantil, além de monitorar e favorecer as condições de saúde e nutrição da criança assistida ${ }^{6}$.

Com relação à cobertura vacinal, podese afirmar que os resultados encontrados (Tabela 5) foram inadequados ao se comparar com estudo realizado em Belo Horizonte, MG, que encontrou $95 \%$ das crianças pesquisadas com as vacinas em dia e devidamente registradas ${ }^{30}$. Sugere-se que os atrasos vacinais e os sub-registros das informações referentes à imunização explicam a deficiência encontrada na cobertura vacinal.

\section{Considerações finais}

Alguns resultados mostraram-se preocupantes ao verificar números insuficientes de práticas assistenciais, sugerindo falhas na prioridade, regularidade e sistematização do atendimento destinado à criança de risco, considerando que o Ministério da Saúde preconiza, através das diretrizes oficiais referentes à atenção às crianças de risco, uma assistência programada e sistematizada, focada no acompanhamento mensal com avaliações do crescimento e desenvolvimento da criança.

Dentre as práticas assistenciais preconizadas, algumas foram insuficientes para o acompanhamento efetivo da criança de risco, como o número de atendimento médico e de enfermagem, visitas domiciliares para os faltosos às consultas mensais programadas e recebimento de orientações específicas sobre promoção a saúde e prevenção de doenças ou agravos.

A descrição das características relacionadas aos aspectos sociodemográficos, biológicos e assistenciais evidenciou a necessidade de se potencializar a atenção ao $\mathrm{RN}$ de risco, especialmente com referência aos aspectos assistenciais. Diante de sua vulnerabilidade à morbimortalidade, os RN de risco necessitam de acesso, prioridade $\mathrm{e}$ garantia de uma assistência efetiva execu- 
tada conforme as preconizações do MS e dos Programas específicos que estreitam a realidade observada com a esperada.

Os grupos expostos a fatores de risco devem ser objeto de atenção especial pelos serviços de saúde, principalmente na atenção primária à saúde, que quais devem organizar estratégias para concentrar esforços dirigidos aos grupos que apresentem maior risco, considerando que a exposição ao risco pode influenciar o padrão saúde-doença da criança levando a um risco maior de morbidade e mortalidade no futuro.

O presente estudo possibilitou conhecer o perfil sociodemográfico, biológico e assistencial do $\mathrm{RN}$ de risco inserido na atenção básica de saúde. A investigação e a descrição dos eventos relacionados ao nascimento e à assistência dispensada à criança de risco durante seu primeiro ano de vida permitiu concluir que as propostas iniciais estabelecidas pelo Ministério da Saúde e pelo próprio PVRNR não se encontram plenamente efetivadas, necessitando de uma reorganização da atenção básica a saúde para assegurar a integralidade da assistência e a continuidade do acompanhamento do desenvolvimento e crescimento biopsicossocial da criança de risco.

\section{Referências}

1. Maran E, Uchimura TT. Mortalidade Neonatal: fatores de risco em um município no sul do Brasil. Rev Eletr Enf 2008; 10 (1): 29-38. Disponível em http://www.fen.ufg. $\mathrm{br} /$ revista/ v10/n1/v10n1a03.htm2008. [Acessado em 25 de setembro de 2009]

2. Slomp FM, Mello DF, Scochi CGS, Leite AM. Assistência ao recém-nascido em um Programa de Saúde da Família. Rev Esc Enferm USP 2007; 41(3): 441-6.

3. Luque ALF. Avaliação da atenção ao recém-nascido de risco no município de Botucatu-SP [dissertação de mestrado]. Botucatu, SP: Universidade Estadual Paulista; 2008.

4. Ministério da Saúde. Secretaria de Atenção á Saúde. Departamento de Ações Programáticas e Estratégicas. Agenda de compromissos para a saúde integral da criança e redução a mortalidade infantil. Brasília, DF, 2004. Disponível em http://www.ministerio.saude. bvs. $\mathrm{br} / \mathrm{html} / \mathrm{pt} / \mathrm{pb}$ _assuntos/saude_crianca.htm [Acessado em 19 de junho de 2008]

5. Ministério da Saúde. Secretaria de Atenção à Saúde. Departamento de Ações Programáticas Estratégicas. Área Técnica de Saúde da Mulher. Pré-natal e puerpério: atenção qualificada e humanizada. Manual técnico: Brasília, DF; 2006.

6. Ministério da Saúde. Secretaria de Políticas de Saúde. Departamento de Atenção Básica. Saúde da Criança: acompanhamento e desenvolvimento infantil. Brasília, DF; 2002.

7. Maringá. Secretaria Municipal de Saúde. Nascer na cidade canção é uma emoção. Rotina - Programa de vigilância ao recém nascido. Apostila, mimeografado. Maringá; 2000.

8. Ministério da Saúde. Secretaria de Atenção Básica. Departamento De Ações Programáticas Estratégicas. Caderneta de Saúde da Criança. $3^{a}$ edição, Brasília, DF; 2007.
9. Phipps MG, Spwers M. Defining early adolescent childbearing. Am J Public Health 2002; 92(1): 125-8.

10. Machado ME. Atenção à saúde prestada ao recémnascido de risco no primeiro ano de vida: um estudo na perspectiva do programa Prá-nenê [dissertação de mestrado]. Porto Alegre, RS: Universidade Federal do Rio Grande do Sul; 2003.

11. Alves AS. Recém-nascido de risco: fatores que contribuem para a precisão de cuidados intensivos neonatais [dissertação de mestrado]. Rio Grande, RS: Universidade Federal do Rio Grande; 2005.

12. Instituto Brasileiro de Geografia e Estatística. Cidades. Disponível em: http://www.ibge.gov.br/cidadesat/ topwindow.htm?1. [Acessado em 27 de março de 2009a]

13. Instituto Brasileiro de Geografia e Estatística [Internet]. Brasília: Ministério do Planejamento, Orçamento e Gestão (BR). Pesquisa Nacional de Amostra Domiciliar (PNAD), 2007. Disponível em http://www.ibge.gov.br/ home/presidencia/noticias/noticia_visualiza. php?id_ noticia $=1230 \& i d \_p a g i n a=1-58 \mathrm{~kg}$. [Acessado em $20 \mathrm{de}$ maio de 2009b].

14. Patella RF. Análise da assistência prestada pelo Programa do Recém Nascido de Risco e sua relação com a hospitalização de menores de um ano e a mortalidade infantil tardia em Santos/SP [dissertação de mestrado]. Campinas, SP: Universidade Estadual de Campinas, Faculdade de Ciências Médicas; 2001.

15. Ministério da Saúde. Centro Brasileiro de Análise e Planejamento. Secretaria de Ciência, Tecnologia e Insumos Estratégicos. Departamento de Ciência e Tecnologia. Pesquisa Nacional sobre Demografia e Saúde da Mulher e da Criança (PNDS) 2006 - Relatório Final. Rio de Janeiro; 2008. 
16. França E, Souza JM, Guimarães MDC, Goulart EMA, Colosimo E, Antunes CMF. Associação entre fatores de risco sócio-econômicos e mortalidade infantil por diarréia, pneumonia e desnutrição em região metropolitana do Sudeste do Brasil: um estudo casocontrole. Cad Saúde Pública 2001; 17(6): 1437-47.

17. Nascimento EMR, Costa MCN, Mota ELA, Paim JS. Estudo de fatores de risco para óbitos de menores de um ano mediante compartilhamento de bancos de dados. Cad Saúde Pública 2008; 24(11): 2593-602.

18. Coimbra LC, Silva AAM, Mochel EG, Alves MTSSB, Ribeiro VS, Aragão VMF et al. Fatores associados à inadequação do uso da assistência pré-natal. Rev Saúde Pública 2003; 37(4): 456-62.

19. Nagahama EEI, Santiago SM. O cuidado pré-natal em hospital universitário: uma avaliação de processo. Cad Saúde Pública 2006; 22(1): 173-9.

20. Ministério da Saúde. DATASUS. Informações de saúde. Estatísticas vitais. [Internet]. Disponível em http:// tabnet.datasus.gov.br/cgi/deftohtm.exe?sinasc/cnv/ nvpr.def. [Acessado em 28 de Abril de 2009]

21. Silva AMR. Fatores de risco para nascimentos pré-termo no Município de Londrina-PR. [tese de doutorado]. São Paulo: Universidade de São Paulo, Faculdade de Saúde Pública; 2008.

22. Manoel CM, Carandina L, Giarola LC. Programa de vigilância ao recém-nascido de risco: ações desenvolvidas no Centro de Saúde Escola de Botucatu, São Paulo. Rev Paul Pediatria 2006; 24(3): 227-32.

23. Isolani FG. Perfil epidemiológico dos nascidos vivos e fatores de risco da mortalidade neonatal no Município de Maringá, PR, no ano de 1995. [monografia de conclusão de curso]. Maringá, PR: Universidade Estadual de Maringá; 1997.
24. World Health Organization. Appropriate technology for birth. Lancet 1985; 2: 436-7.

25. Serafim D. Mortalidade neonatal em Maringá - PR, 1997-2000 [tese de doutorado]. São Paulo: Universidade de São Paulo, Faculdade de Saúde Pública; 2002.

26. Brandt I, Sticker EJ, Galsche R, Lentze MJ. Catch-up growth of supine length/height of very low birth weight, small for gestational age preterm infants to adulthood. $J$ Pediatr 2005; 147(5): 662-8.

27. Cunha AA, Fernandes DS, Melo PF, Guedes MH. Fatores associados à asfixia perinatal. Rev Bras Ginecol Obstet 2004; 26(10): 799-805.

28. Puccini RF, Silva NN, Araújo NS, Pedroso GC, Silva EMK. Saúde infantil: condições de vida e utilização de serviços de saúde em área da Região Metropolitana de São Paulo, 1996. Rev Bras Saúde Matern Infant 2002; 2(2): 143-55.

29. Carvalho MR, Tamez RN. Amamentação: bases científicas para a prática profissional. Rio de Janeiro: Ed. Guanabara Koogan; 2002. p. 452.

30. Alves CRL, Lasmar LMLBF, Goulart LMHF, Alvim CG, Maciel GVR, Viana MRA et al. Qualidade do preenchimento da Caderneta de Saúde da Criança e fatores associados. Cad Saúde Pública 2009; 25(3): 58395.

Recebido em: 23/01/10

Versão final reapresentada em: 07/01/11

Aprovado em: 28/02/11 